EUROPEAN ORGANIZATION FOR NUCLEAR RESEARCH

CERN-EP-2000-012

February 4, 2000

\title{
Dimuon and charm production in nucleus-nucleus collisions at the CERN-SPS
}

\author{
NA38 and NA50 Collaborations
}

M.C. Abreu ${ }^{7, a)}$, B. Alessandro ${ }^{12)}$, C. Alexa ${ }^{4)}$, R. Arnaldi ${ }^{12)}$, J. Astruc ${ }^{9)}$, M. Atayan ${ }^{14)}$, C. Baglin ${ }^{2)}$, A. Baldit ${ }^{3)}$, M. Bedjidian ${ }^{13)}$, F. Bellaiche ${ }^{13)}$, S. Beolè ${ }^{12)}$, V. Boldea ${ }^{4)}$, P. Bordalo ${ }^{7, b)}$, A. Borhani ${ }^{10)}$, A. Bussière ${ }^{2)}$, L. Capelli ${ }^{13)}$, V. Capony $^{2)}$, L. Casagrande ${ }^{7)}$, J. Castor ${ }^{3)}$, T. Chambon ${ }^{3)}$, B. Chaurand ${ }^{10)}$, I. Chevrot ${ }^{3)}$, B. Cheynis ${ }^{13)}$, E. Chiavassa ${ }^{12)}$, C. Cicalo ${ }^{5)}$, T. Claudino ${ }^{7)}$, M.P. Comets ${ }^{9)}$, N. Constans ${ }^{10)}$, S. Constantinescu ${ }^{4)}$, J. Cruz ${ }^{7)}$, A. De Falco ${ }^{5)}$, N. De Marco ${ }^{12)}$, G. Dellacasa ${ }^{1)}$, A. Devaux $^{3)}$, S. Dita $^{4)}$, O. Drapier ${ }^{13,6)}$, L. Ducroux ${ }^{13)}$, B. Espagnon ${ }^{3)}$, J. Fargeix ${ }^{3)}$, F. Fleuret ${ }^{10)}$, P. Force $^{3)}$, M. Gallio $^{12)}$, Y.K. Gavrilov ${ }^{8)}$, C. Gerschel ${ }^{9)}$, P. Giubellino ${ }^{12)}$, M.B. Golubeva ${ }^{8)}$, M. Gonin ${ }^{10)}$, A.A. Grigorian ${ }^{14)}$, J.Y. Grossiord ${ }^{13)}$, F.F. Guber ${ }^{8)}$, A. Guichard ${ }^{13)}$, H. Gulkanyan ${ }^{14)}$, R. Hakobyan ${ }^{14)}$, R. Haroutunian ${ }^{13)}$, M. Idzik ${ }^{12, c)}$, D. Jouan ${ }^{9)}$, T.L. Karavitcheva ${ }^{8)}$, L. Kluberg ${ }^{10)}$, A.B. Kurepin ${ }^{8)}$, Y. Le Bornec ${ }^{9)}$, C. Lourenço ${ }^{6)}$, P. Macciotta ${ }^{5)}$, M. Mac Cormick $^{9)}$, A. Marzari-Chiesa ${ }^{12)}$, M. Masera ${ }^{12)}$, A. Masoni ${ }^{5)}$, S. Mehrabyan ${ }^{14)}$, M. Monteno ${ }^{12)}$, S. Mourgues ${ }^{3)}$, A. Musso ${ }^{12)}$, F. Ohlsson-Malek ${ }^{13, d)}$, P. Petiau ${ }^{10)}$, A. Piccotti ${ }^{12)}$, J.R. Pizzi ${ }^{13)}$, G. Puddu ${ }^{5)}$, C. Quintans ${ }^{7}$, C. Racca ${ }^{11)}$, L. Ramello ${ }^{1)}$, S. Ramos ${ }^{7, b)}$, P. Rato-Mendes ${ }^{7)}$, L. Riccati ${ }^{12)}$, A. Romana ${ }^{10)}$, I. Ropotar ${ }^{6)}$, P. Saturnini ${ }^{3)}$, E. Scomparin ${ }^{12, *)}$, S. Serci ${ }^{5)}$, R. Shahoyan ${ }^{7, e)}$, S. Silva ${ }^{7)}$, M. Sitta ${ }^{1)}$, C. Soave ${ }^{12)}$, P. Sonderegger ${ }^{6, b)}$, X. Tarrago ${ }^{9)}$, N.S. Topilskaya ${ }^{8)}$, G.L. Usai ${ }^{5)}$, E. Vercellin ${ }^{12)}$, L. Villatte ${ }^{9)}$, N. Willis ${ }^{9)}$

\begin{abstract}
Muon pair production in p-A, $\mathrm{S}-\mathrm{U}$ and $\mathrm{Pb}-\mathrm{Pb}$ collisions has been studied by the NA38 and NA50 collaborations at the CERN SPS. In this paper we present an analysis of the dimuon invariant mass region between the $\phi$ and the $\mathrm{J} / \psi$. After subtraction of the combinatorial background due to $\pi$ and $\mathrm{K}$ decays, we find that the superposition of dimuons from semi-leptonic decays of D mesons and from the Drell-Yan process reproduces well the mass and transverse momentum spectra measured in $\mathrm{p}-\mathrm{A}$ collisions. From the yield of open charm dimuons required to fit our data we extract a charm production cross section in good agreement with previous measurements. A linear extrapolation of the $\mathrm{p}-\mathrm{A}$ sources with the product of the mass numbers of the projectile and target nuclei, $A \times B$, underestimates the yield measured in $\mathrm{S}-\mathrm{U}$ and $\mathrm{Pb}-\mathrm{Pb}$ collisions. The excess increases with the number of participant nucleons, and the ratio between the observed dimuon yield and the expected sources reaches a factor 2 for central $\mathrm{Pb}-\mathrm{Pb}$ interactions. The kinematical distributions of the measured dimuon excess are compatible with those expected from the open charm contribution.
\end{abstract}

Accepted by Euro. Phys. J. C 
1) Università del Piemonte Orientale, Alessandria and INFN-Torino, Italy

2) LAPP, CNRS-IN2P3, Annecy-le-Vieux, France.

3) LPC, Univ. Blaise Pascal and CNRS-IN2P3, Aubière, France.

4) IFA, Bucharest, Romania.

5) Università di Cagliari/INFN, Cagliari, Italy.

6) CERN, Geneva, Switzerland.

7) LIP, Lisbon, Portugal.

8) INR, Moscow, Russia.

9) IPN, Univ. de Paris-Sud and CNRS-IN2P3, Orsay, France.

10) LPNHE, Ecole Polytechnique and CNRS-IN2P3, Palaiseau, France.

11) IRS, Univ. Louis Pasteur and CNRS-IN2P3, Strasbourg, France.

12) Università di Torino/INFN, Torino, Italy.

13) IPN, Univ. Claude Bernard and CNRS-IN2P3, Villeurbanne, France.

14) YerPhI, Yerevan, Armenia.

a) Also at UCEH, Universidade do Algarve, Faro, Portugal

b) Also at IST, Universidade Técnica de Lisboa, Lisbon, Portugal

c) Now at FPNT, University of Mining and Metallurgy, Cracow, Poland

d) Now at ISN, Univ. Joseph Fourier and CNRS-IN2P3, Grenoble, France

e) On leave of absence from YerPhI, Yerevan, Armenia

*) Corresponding author: Enrico Scomparin, scomparin@to.infn.it 


\section{Introduction}

It is widely recognized that the study of dilepton production plays a very important role in the understanding of ultrarelativistic heavy-ion physics [1]. In particular, dileptons have the very interesting property of not suffering strong interactions with the surrounding hadronic medium they traverse after being produced.

An experiment aimed at detecting dilepton production in heavy-ion collisions can access a large variety of physics observables, many of them sensitive to the possible formation of a deconfined state of matter (the so-called Quark-Gluon Plasma, or QGP) or allowing to characterize the thermodynamical state of the produced matter.

The experimental challenges are mainly due to the small production cross sections (compared to hadron production) and to the partial overlap in phase space of the various dilepton sources. The dilepton invariant mass spectrum appears as a wide continuum dominated by the resonance $\left(\rho, \omega, \phi, \mathrm{J} / \psi, \psi^{\prime}\right)$ decay peaks. While the contribution of the latter can be rather easily singled out, the deconvolution of the processes in the continuum is not trivial. This problem plays a crucial role in the analysis presented in this paper, dedicated to the study of the dimuon mass spectrum in the region between the $\phi$ and the $\mathrm{J} / \psi$, known as the Intermediate Mass Region (IMR). This zone of the mass spectrum is particularly interesting since it may contain dileptons created in the thermalized QGP or hadron gas produced in nucleus-nucleus collisions (the so-called thermal dimuons) [2]. Furthermore, the semi-leptonic decay of charmed hadron pairs is known to produce a sizeable signal in the IMR; the study of this mass window, therefore, allows to perform an indirect measurement of open charm production in heavy ion interactions.

This paper presents results obtained with data measured in $\mathrm{p}-\mathrm{A}(\mathrm{A}=\mathrm{Al}, \mathrm{Cu}, \mathrm{Ag}$, $\mathrm{W}), \mathrm{S}-\mathrm{U}$ and $\mathrm{Pb}-\mathrm{Pb}$ collisions, in the NA38/NA50 experiments at the CERN SPS. Previous investigations, performed by the NA38 [3, 4], HELIOS/3 [5] and NA50 [6] collaborations, have shown that the dimuon production in nucleus-nucleus collisions is abnormally enhanced with respect to proton-nucleus interactions.

The aim of this analysis, besides confirming the previous NA38/NA50 observations, is to investigate in more detail the centrality dependence of the dimuon yield and to test against the data some explanations of the observed excess. The improvements are due to the much larger data sample available now and to an upgraded analysis technique.

The paper is organized as follows. After a brief summary of the experimental setup and data taking conditions, we will present in detail the combinatorial background subtraction technique. We will then proceed to the analysis of the p-A data samples, disentangling the various dimuon sources contributing to the mass spectrum. Finally, the nucleus-nucleus data will be studied, by comparing the measured spectra with the expected extrapolation of the p-A results. Possible explanations of the observed anomaly will also be discussed. 


\section{Apparatus and data taking conditions}

The data analyzed in this paper have been collected with two experimental set-ups. The measurement of dimuon production in $\mathrm{S}-\mathrm{U}$ collisions has been carried out using the NA38 apparatus [7], which consisted of a muon spectrometer [8], covering the rapidity domain $3<y_{\text {lab }}<4$, with a toroidal air-gap magnet, two sets of four MWPCs and four trigger hodoscopes, made of plastic scintillators. The hadrons produced in the collision were absorbed in a $4.8 \mathrm{~m}$ long carbon dump, with a central $\mathrm{W} / \mathrm{U}$ plug to absorb the beam particles which did not interact in the target. The centrality of the collision was estimated measuring the transverse neutral energy $E_{\mathrm{T}}$ in the pseudorapidity domain $1.7<\eta<4$.1. A Pb-scintillating fibers electromagnetic calorimeter was used for this purpose. A segmented target was adopted in order to maximize the number of events, while minimizing unidentified reinteractions of nuclear fragments. A set of scintillators, surrounding the target region, allowed to determine the vertex position and to identify reinteractions, which could bias the centrality measurement. The luminosity was monitored by means of a beam hodoscope, made of plastic scintillators, which counted the incident sulphur ions.

The NA50 apparatus [9], sketched in Fig. 1, has been designed for the study of $\mathrm{Pb}-\mathrm{Pb}$ collisions, where higher trigger rates and radiation levels are reached. It has inherited the NA38 muon spectrometer, while the target region, where the highest radiation levels ( $\sim 1 \mathrm{Grad} / \mathrm{month})$ are attained, has been substantially upgraded. The beam hodoscope and target detectors [10] have been rebuilt using quartz blades instead of plastic scintillators. Furthermore, the centrality detection has become more sophisticated; besides the electromagnetic calorimeter, now covering the domain $1.1<\eta<2.3$, NA50 is equipped with a Si-strip charged multiplicity detector [11] and a zero-degree calorimeter (ZDC) [12], made of quartz fibres embedded in tantalum. This device, centered on the beam axis, is located inside the hadron absorber, and measures the energy $E_{\mathrm{ZDC}}$ of the spectator nucleons from the projectile.

The trigger of the experiment is given by a coincidence, in the scintillator hodoscopes, of two charged tracks. Each track is defined by means of hit patterns in the four trigger hodoscopes, corresponding to muons produced in the target region. The trigger efficiency is measured with a system of two hodoscopes, specially designed for this purpose. Finally, a new acquisition system was built, in order to face the very high trigger rates $[13,14]$.

The p-A data samples analyzed in this paper have also been collected with the NA50 experimental apparatus. For such runs a passive target has been used. The centrality detectors were not used, since for p-A collisions $E_{\mathrm{T}}, E_{\mathrm{ZDC}}$ and the charged multiplicity are very loosely correlated with the geometry of the collision. The luminosity measurement has been performed by means of a set of ionization chambers.

Concerning the data taking conditions, the p-A interactions have been studied with an incident beam intensity of about $3 \cdot 10^{9} \mathrm{p} /$ burst, in a $2.5 \mathrm{~s}$ spill, with target thicknesses ranging from 0.3 to $0.5 \lambda_{\mathrm{I}}$. For $\mathrm{S}-\mathrm{U}$ the experiment has been run at about $8 \cdot 10^{7}$ incident ions/burst, with a $5 \mathrm{~s}$ spill and $12 \mathrm{U}$ subtargets; the total target 
thickness was $0.2 \lambda_{\mathrm{I}}$. Finally, in the study of $\mathrm{Pb}-\mathrm{Pb}$ collisions, the average beam intensity was $5 \cdot 10^{7}$ ions/burst, with a $5 \mathrm{~s}$ spill and $7 \mathrm{~Pb}$ subtargets with a total thickness of $0.3 \lambda_{\mathrm{I}}$. The average magnetic field in the toroidal magnet was $1.2 \mathrm{Tm}$ for the NA38 set-up (S-U data) and 2.1 Tm for NA50 (p-A and Pb-Pb data), resulting in a different acceptance for low $m_{\mathrm{T}}$ dimuons $\left(m_{\mathrm{T}}^{\min } \sim 0.9 \mathrm{GeV} / \mathrm{c}^{2}\right.$ for NA38 and $1.3 \mathrm{GeV} / \mathrm{c}^{2}$ for NA50).

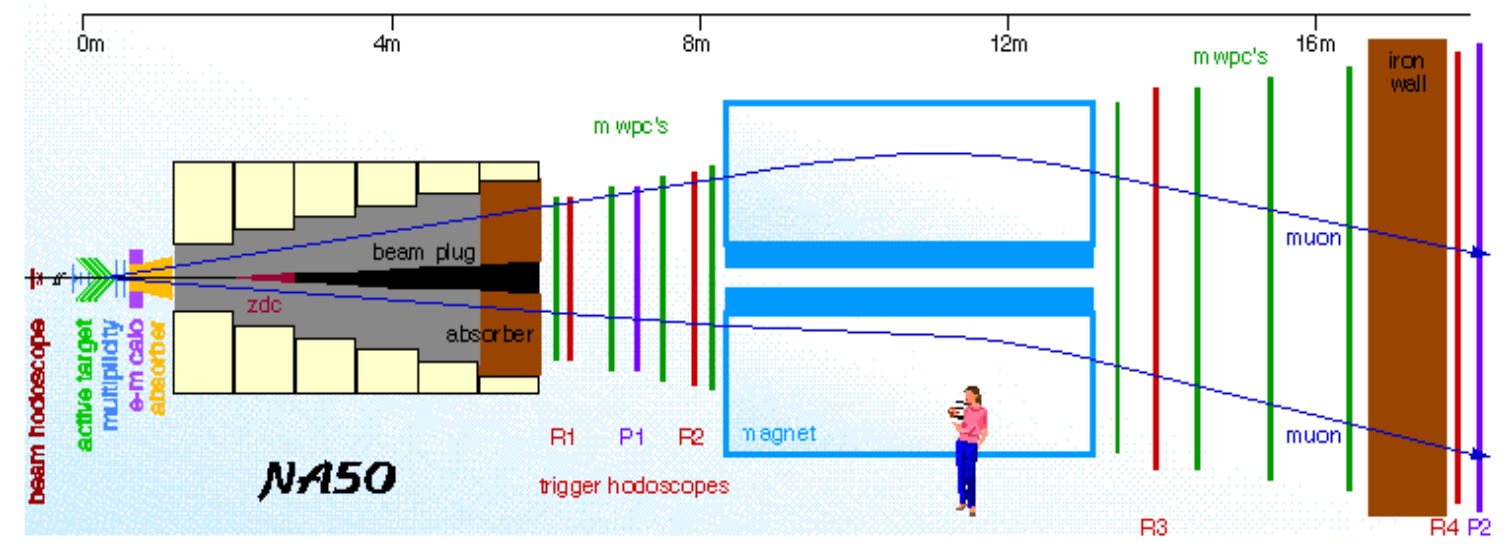

Figure 1: The NA50 experimental apparatus.

\section{Data reduction and centrality selection}

The standard NA38/NA50 offline data reduction procedure has been adopted $[9,15]$. It essentially requires to have two tracks fully reconstructed in the muon spectrometer, pointing to the target.

For $\mathrm{S}-\mathrm{U}$ and $\mathrm{Pb}-\mathrm{Pb}$ collisions the assignment of the subtarget where the interaction takes place is performed by means of the target detectors. They detect the particles emitted in the nucleus-nucleus collisions, allowing to determine the primary interaction vertex. The event is retained if such a vertex is identified and no secondary vertices, due to fragment reinteractions, are detected.

For p-A interactions, the vertex is assigned to the centre of the (single) target. This approximate determination of the collision vertex does not affect very much the determination of the dimuon kinematical variables. For instance, the mass resolution in the $\mathrm{J} / \psi$ region is about $120 \mathrm{MeV}$ for $\mathrm{p}-\mathrm{W}$ collisions and $98 \mathrm{MeV}$ for $\mathrm{Pb}-\mathrm{Pb}$ collisions, when the active target selection is used, and $\sim 105 \mathrm{MeV}$ if not. To study nucleus-nucleus interactions it is very interesting to access the widest possible range of centralities. However, the efficiency of the active target detector is rather low for peripheral events. Therefore, we have chosen to carry out the $\mathrm{Pb}-\mathrm{Pb}$ analysis without the active target selection and we have defined as on-target the events lying in the narrow $E_{\mathrm{T}}$ vs $E_{\mathrm{ZDC}}$ correlation area (see Ref. [15] for details). 
Various quality cuts are then applied to the event sample. Basically, a set of halo detectors helps rejecting off-target events, while the beam hodoscope recognizes events with two or more ions incident within a 20 ns time window. Such events could produce piled-up interactions, leading to a bias in the measurement provided by the centrality detectors, and are therefore tagged for offline rejection. To avoid systematic effects, runs have been rejected when departing from stability criteria related to beam quality and/or apparatus performance.

Finally, in order to discard dimuons produced at the edges of the spectrometer's acceptance, the cuts $0<y_{\mathrm{cm}}<1$ and $-0.5<\cos \theta_{C S}<0.5$ have been imposed in the study of $\mathrm{S}-\mathrm{U}$ and $\mathrm{Pb}-\mathrm{Pb}$ collisions. The angle $\theta_{C S}$ is the polar angle of the muons relative to the beam axis in the rest frame of the dimuon (the so-called Collins-Soper reference frame). For p-A interactions, the higher incident energy shifts the center of mass rapidity by about half unit. Therefore, the selection $-0.52<y_{\mathrm{cm}}<0.48$ has been adopted, besides the above mentioned $\theta_{C S}$ cut.

\begin{tabular}{ccccr}
\hline & $E_{\mathrm{T}}$ bin & $\Delta E_{\mathrm{T}}(\mathrm{GeV})$ & $\langle b\rangle(\mathrm{fm})$ & \multicolumn{1}{c}{$\left\langle N_{\text {part }}\right\rangle$} \\
\hline \multirow{4}{*}{$\mathrm{S}-\mathrm{U}$} & 1 & $13-34$ & 7.6 & $43 \pm 7$ \\
& 2 & $34-50$ & 6.0 & $65 \pm 6$ \\
& 3 & $50-64$ & 4.8 & $84 \pm 5$ \\
& 4 & $64-77$ & 3.6 & $101 \pm 5$ \\
& 5 & $77-88$ & 1.9 & $116 \pm 3$ \\
\hline \multirow{4}{*}{$\mathrm{Pb}-\mathrm{Pb}$} & 1 & $<20$ & $>9.9$ & $63 \pm 18$ \\
& 2 & $20-32$ & 9.3 & $110 \pm 11$ \\
& 3 & $32-45$ & 8.3 & $149 \pm 11$ \\
& 5 & $45-57$ & 7.3 & $189 \pm 12$ \\
& 6 & $57-69$ & 6.3 & $231 \pm 13$ \\
& 7 & $89-81$ & 5.5 & $273 \pm 11$ \\
& 8 & $93-105$ & 3.5 & $310 \pm 10$ \\
& 9 & $>105$ & $<3.3$ & $381 \pm 11$ \\
\hline
\end{tabular}

Table 1: $E_{\mathrm{T}}$ classes used in the analysis, with the corresponding average values of the impact parameter, $\langle b\rangle$, and of the number of participants, $\left\langle N_{\text {part }}\right\rangle$. The error bars on $\left\langle N_{\text {part }}\right\rangle$ represent the r.m.s. of the distribution in each $E_{\mathrm{T}}$ bin.

The $\mathrm{S}-\mathrm{U}$ and $\mathrm{Pb}-\mathrm{Pb}$ event samples have been divided in centrality classes according to the measured neutral e.m. energy, $E_{\mathrm{T}}$. We show in Table 1 the average impact parameter, $\langle b\rangle$, and average number of participant nucleons, $\left\langle N_{\text {part }}\right\rangle$, corresponding to each $E_{\mathrm{T}}$ class. These quantities have been evaluated with a Glauber model description of the nucleus-nucleus collision (see Ref. [16] for details); the nuclear density profiles have been described using the standard three-parameter Woods-Saxon form. The relationship between $N_{\text {part }}$ and $b$ has been further checked using the VENUS 4.12 event generator [17], which gives the same correlation, within errors. 


\section{The evaluation of the combinatorial background}

The dominant dimuon source in the IMR is the 'combinatorial background' due to the decays of $\pi$ and $\mathrm{K}$ mesons. In the NA38/NA50 data taking conditions it is found to account for between $85 \%$ ( $\mathrm{p}$-A, peripheral nucleus-nucleus collisions) and $95 \%$ (central $\mathrm{Pb}-\mathrm{Pb}$ collisions) of the total opposite sign dimuon yield. Its proper evaluation is therefore crucial for a correct estimate of the other well known IMR physics sources, i.e. the Drell-Yan mechanism $\left(q \bar{q} \rightarrow \mu^{+} \mu^{-}\right)$and the semi-leptonic decay $(D \rightarrow \mu X)$ of charmed meson pairs.

Several methods exist for the treatment of the combinatorial background in a dilepton experiment. All of them make use of the measured sample of like-sign lepton pairs, which is entirely due to $\pi$ and K decays. Simple probability arguments link the differential distribution of opposite sign background dimuons $\mathrm{d} N_{\mathrm{bck}}^{+-} / \mathrm{d} X$ (where $\left.X=M, p_{\mathrm{T}}, y, \cos \theta\right)$ to the corresponding like-sign distributions. If the background muons falling in the acceptance of the apparatus come from totally uncorrelated parent mesons, the basic relation

$$
\frac{\mathrm{d} N_{\mathrm{bck}}^{+-}}{\mathrm{d} X}=2 \sqrt{\frac{\mathrm{d} N^{++}}{\mathrm{d} X} \frac{\mathrm{d} N^{--}}{\mathrm{d} X}} \cdot \frac{A^{+-}}{\sqrt{A^{++} A^{--}}}
$$

holds, where $A^{+-}, A^{++}, A^{--}$, are the acceptances for opposite-sign and like-sign dimuons. An offline cut is applied to the data in order to ensure that the acceptance of the spectrometer is independent of the charge of the detected muons; consequently we get $A^{+-} / \sqrt{A^{++} A^{--}}=1$.

This treatment of the background faces two potential problems, which are outlined hereafter and discussed in more detail in the following subsections. First, it is based on the assumption that the meson production is completely charge uncorrelated. Strictly speaking this is true only if the multiplicity of charged particles generated in the interaction is so large that the existing physical charge correlation, due to the total charge conservation, is smeared out. This is certainly the case for central $\mathrm{Pb}-\mathrm{Pb}$ collisions, where the charged multiplicity in the acceptance of the spectrometer reaches a value of $\sim 400$ [18]. For peripheral nucleus-nucleus collisions, where the multiplicities are almost one order of magnitude lower, as well as in p-nucleus interactions, where the multiplicities are even lower, the charge correlation effects will play a role. To account for these effects, the right hand side of Eq. (1) has to be multiplied by a factor $R$.

The second problem concerns the error on the determination of the background shape. Clearly, when the combinatorial background is large, as in $\mathrm{Pb}-\mathrm{Pb}$ collisions, its shape must be known with a high degree of accuracy in order to induce the smallest possible errors on the background subtracted spectra. The necessary accuracy can be reached using the Fake Opposite Sign (FOS) method, a mixed event technique described in Ref. [19], which allows to build the expected combinatorial background shape making use of the measured single muon distributions from the like-sign samples. 


\subsection{The charge correlation effects}

The charge correlation effects, parametrized by the introduction of a multiplicative factor $R$ in Eq.(1), have been evaluated by means of a Monte-Carlo simulation. Using the VENUS 4.12 event generator, a large number $\left(>10^{5}\right)$ of $\mathrm{p}-\mathrm{A}, \mathrm{S}-\mathrm{U}$ and $\mathrm{Pb}-\mathrm{Pb}$ events, at the relevant energies, have been generated, and the $\pi$ and $\mathrm{K}$ four-momenta have been stored. For each meson $j$ of charge $a$ the total decay probability $P_{j}^{a}$ in the NA38/NA50 experimental set-up has been calculated, taking into account the energy loss along its flight path inside the hadron absorber. Next, for each event, the generated mesons have been combined in all possible ways and each meson has been forced to decay to a muon. For each meson-meson combination we get a dimuon, and compute its kinematical variables $\left(M, p_{\mathrm{T}}, y, \cos \theta\right)$. To each dimuon is assigned a weight equal to the product of the decay probabilities of its parents,

$$
P_{j k}^{a b}=P_{j}^{a} P_{k}^{b}
$$

For each system under study, the $R$ parameter in a generic kinematical domain $\mathcal{F}$ is given by

$$
R(\mathcal{F})=\frac{\sum_{n=1}^{N_{V}} \sum_{j, k} P^{+-}(\mathcal{F})_{j, k}}{2 \sqrt{\left(\sum_{n=1}^{N_{V}} \sum_{j, k} P^{++}(\mathcal{F})_{j, k}\right) \times\left(\sum_{n=1}^{N_{V}} \sum_{j, k} P^{--}(\mathcal{F})_{j, k}\right)}}
$$

where $N_{V}$ is the total number of generated events and the sum $\sum_{j, k}$ runs on the dimuons produced for each event in the domain $\mathcal{F}$. In Fig. 2 we show $R$ as a function of the dimuon transverse momentum, for $\mathrm{p}-\mathrm{W}$ collisions at $450 \mathrm{GeV} / \mathrm{c}$. We note that $R$ is larger than one. As expected, in the low-multiplicity p-A collisions, the charge correlation between the parent mesons cannot be neglected. Moreover, the clear dependence of $R$ on $p_{T}$ indicates that the charge correlation effects are a function of the kinematical domain where the dimuon measurement is performed. Generally, for a given system, $R$ is found to increase both with $y$ and $p_{\mathrm{T}}$. As a consequence, in our data analysis $R$ must be calculated for the domain $\mathcal{D}$, defined by the cuts $0<y_{\mathrm{cm}}<1\left(-0.52<y_{\mathrm{cm}}<0.48\right.$ for $\left.\mathrm{p}-\mathrm{A}\right)$ and $-0.5<\cos \theta_{C S}<0.5$, corresponding to the acceptance window of the apparatus. Within this domain, each dimuon has been further weighted with its detection probability in the spectrometer, which, for IMR dimuons, is peaked in a relatively narrow region in $y, \cos \theta_{C S}$ and $\mathrm{m}_{T}$.

In such a region the $R$ factors have been found to vary very little; therefore we can use a constant value of $R$ for each of the systems under study. However, in nucleusnucleus collisions, where we deal with various centrality classes, it is necessary to take into account a possible dependence of $R$ on the impact parameter $b$. This dependence has been investigated and parametrised by generating samples of events for discrete values of $b$. In Fig. 3 we show $R$ vs $b$ for $\mathrm{S}-\mathrm{U}$ and $\mathrm{Pb}-\mathrm{Pb}$ collisions, where a clear departure from $R=1$ can be seen for peripheral events.

The $R$ value corresponding to each of the centrality classes used in the analysis is then readily obtained as a weighted average of $R(b)$ over the $b$ distribution of the 


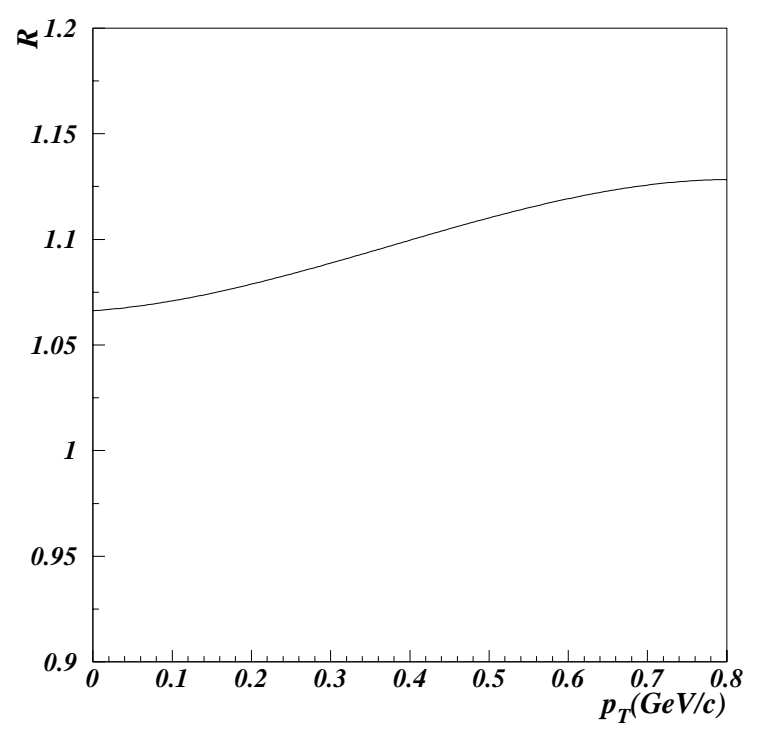

Figure 2: $R$ as a function of the dimuon $p_{\mathrm{T}}$, calculated for $\mathrm{p}-\mathrm{W}$ collisions before applying any kinematical selection.

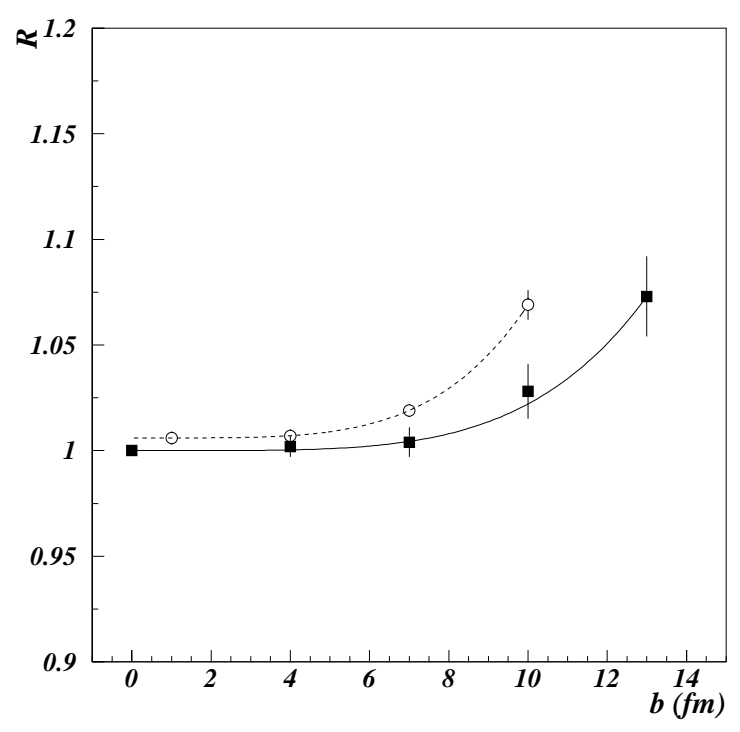

Figure 3: $R$ as a function of $b$ for S-U (circles) and $\mathrm{Pb}-\mathrm{Pb}$ (squares) collisions, before applying any kinematical selection.

\begin{tabular}{ccl}
\hline System & $E_{\mathrm{T}}$ bin & $R-1(\%)$ \\
\hline & 1 & $3.5 \pm 1.1$ \\
& 2 & $1.7 \pm 0.3$ \\
& 3 & $1.0 \pm 0.2$ \\
& 4 & $0.6 \pm 0.1$ \\
$\mathrm{~Pb}-\mathrm{Pb}$ & 5 & $0.3 \pm 0.06$ \\
& 6 & $0.1 \pm 0.03$ \\
& 7 & $0.1 \pm 0.02$ \\
& 8 & $0.1 \pm 0.01$ \\
& 9 & $0.0 \pm 0.01$ \\
\hline & 1 & $2.3 \pm 0.5$ \\
$\mathrm{~S}-\mathrm{U}$ & 2 & $1.3 \pm 0.2$ \\
& 3 & $0.9 \pm 0.07$ \\
& 4 & $0.7 \pm 0.04$ \\
& 5 & $0.6 \pm 0.01$ \\
\hline
\end{tabular}

Table 2: $R$ factors for the $9 \mathrm{~Pb}-\mathrm{Pb}$ and $5 \mathrm{~S}-\mathrm{U}$ centrality bins, in the phase space domains of the data analysis. 
events in that class. The calculated values, for $\mathrm{Pb}-\mathrm{Pb}$ and $\mathrm{S}-\mathrm{U}$ collisions, are collected in Table 2.

The use of high beam intensities, with the consequent pile-up of incident projectiles, plays an important role in the determination of $R$. The nucleus-nucleus data sample is essentially pile-up free because of the beam hodoscope cut. On the contrary, in p-A collisions such a device cannot be used because of the very high beam intensity ( $I \sim 10^{9} \mathrm{p} / \mathrm{s}$ ) and the secondaries from several p-A interactions can overlap in the detector readout gate. Basically, if there is pile-up, a combinatorial background event can be generated by muons produced in different interactions; the overall effect is the smearing of the charge correlations between the parent mesons, which in this case are uncorrelated by definition.

In our calculation the pile-up is simulated assuming a Poisson distribution for the incident protons hitting the target in the 20 ns readout gate. The number of interacting protons for each trigger is computed, taking into account the target thickness. Every meson is then combined with all the other mesons produced in the $N_{p u}$ interactions and $R$ is calculated on this larger, and less charge correlated, sample. In Table 3 we show the values of $R$, in the restricted kinematical window of the analysis, obtained at the nominal beam intensities. The values corresponding to the low beam intensity limit (no pile-up) are also shown.

\begin{tabular}{ccc}
\hline System & $\begin{array}{c}R \pm \sigma_{R} \\
\text { (low intensity) }\end{array}$ & $\begin{array}{c}R \pm \sigma_{R} \\
\left(I=2.5 \cdot 10^{9} \mathrm{p} / \text { burst }\right)\end{array}$ \\
\hline $\mathrm{p}-\mathrm{Al}$ & $1.19 \pm 0.05$ & $1.06 \pm 0.02$ \\
$\mathrm{p}-\mathrm{Cu}$ & $1.26 \pm 0.04$ & $1.06 \pm 0.02$ \\
$\mathrm{p}-\mathrm{Ag}$ & $1.21 \pm 0.04$ & $1.06 \pm 0.01$ \\
$\mathrm{p}-\mathrm{W}$ & $1.21 \pm 0.03$ & $1.05 \pm 0.01$ \\
\hline
\end{tabular}

Table 3: $R$ factors for the p-A systems at $450 \mathrm{GeV} / \mathrm{c}$.

Finally, we must emphasize that the estimate of $R$ outlined in this section relies on the use of a Monte-Carlo simulation. It is therefore important to compare the results with a measured value of $R$. Such a measurement is indeed possible, and has been performed by NA38 using two sets of data, relative to $\mathrm{p}-\mathrm{W}$ interactions at $200 \mathrm{GeV} / \mathrm{c}$, collected with two different hadron absorber set-ups. Since the two sets of data can only differ in their background component, while the other dimuon sources have to be the same, it is possible, by means of a simultaneous fit of the two dimuon mass distributions, to estimate $R$ (see Ref. [20] for details). The $R$ factor obtained in that analysis, $R_{\text {meas }}^{p W, 200 ~ G e V}=1.19 \pm 0.04$, is nicely reproduced by a calculation of the same quantity performed with the procedure described in this section, which gives $R^{p W, 200 \mathrm{GeV}}=1.21 \pm 0.02$. 


\subsection{The FOS method}

The Fake Opposite Sign method, also known as the method of exhaustive combinations, has been used to determine the shape of the combinatorial background for $\mathrm{Pb}-\mathrm{Pb}$ collisions. In this situation, because of the particularly high background levels, it is necessary to reduce as much as possible the size of the associated errors.

The $\mathrm{Pb}-\mathrm{Pb}$ data set has been divided into subsamples corresponding to the 9 centrality bins used in the analysis. Within each subsample, the single muon distributions have been extracted from the like-sign dimuon events. Then, all the possible opposite sign dimuons have been generated by combining the muons into pairs. The dimuon differential distributions calculated in this way agree with the ones derived using Eq. (1) but, since each muon is combined several times with other different muons, the error is reduced (see Ref. [21] for details). A comparison of the results obtained with the two methods can be seen in Fig. 4. The error on the background shape is reduced by a factor $\sim 2$ when the FOS technique is used. Furthermore, the background subtracted spectrum is found to be smoother. It has to be noted that this method gives only the shape of the background; the absolute normalization is still given by Eq. (1), with the appropriate $R$ factor.

\section{Analysis}

\section{1 p-A collisions}

The contributions of the IMR physics sources have been unfolded by means of a fit to the opposite sign dimuon mass spectra. The fitting function has been chosen as a sum of the shapes of the known sources, with free relative normalization factors. The region beyond the $\mathrm{J} / \psi$ mass has been included in the fit, in order to constrain the contribution of the Drell-Yan process. The region $M>1.5 \mathrm{GeV} / \mathrm{c}^{2}$ of the opposite sign p-A spectra has been described by the function

$$
\frac{\mathrm{d} N}{\mathrm{~d} M}^{+-}=R \frac{\mathrm{d} N}{\mathrm{~d} M}^{\mathrm{bck}}+n^{D \bar{D}} \frac{\mathrm{d} N}{\mathrm{~d} M}^{D \bar{D}}+n^{D Y} \frac{\mathrm{d} N^{D Y}}{\mathrm{~d} M}+n^{\psi} \frac{\mathrm{d} N}{\mathrm{~d} M}+n^{\psi^{\prime}} \frac{\mathrm{d} N^{\psi^{\prime}}}{\mathrm{d} M} .
$$

The contribution of the combinatorial background, $R \mathrm{~d} N^{\mathrm{bck}} / \mathrm{d} M$, has been fixed with the procedure described in the previous sections. The shapes $\mathrm{d} N^{i} / \mathrm{d} M$ of the other dimuon sources have been evaluated by means of a Monte-Carlo simulation. More in detail, the mass distributions for $D Y$ and open charm $(D \bar{D})$ decays have been calculated using the event generator PYTHIA 5.7 [22], with the MRS A set of parton distribution functions [23], $m_{c}=1.35 \mathrm{GeV} / \mathrm{c}^{2}$ and $\left\langle k_{\mathrm{T}}^{2}\right\rangle=0.8(\mathrm{GeV} / \mathrm{c})^{2}$. We have verified that the calculated mass distributions are not very sensitive to the specific values of these parameters [24]. The charmonia resonances have been generated using phenomenological $y$ and $p_{\mathrm{T}}$ distributions, and a flat $\cos \theta_{C S}$ decay distribution (see Ref. [16] for details). The generation has been done in a kinematical region larger than the one covered by the experiment, to account for smearing effects. The dimuons 

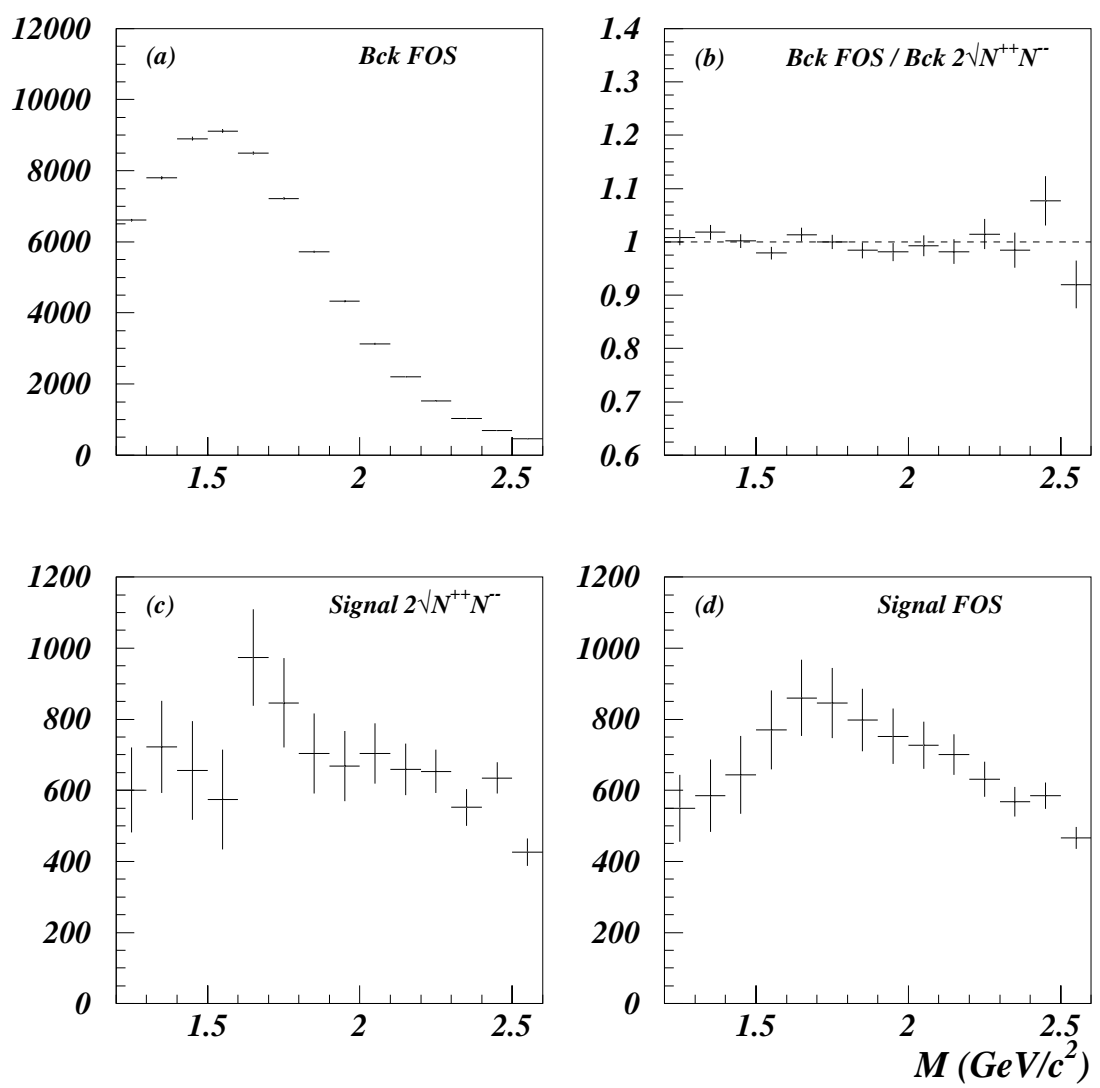

Figure 4: Comparison between the two background calculations, in the second centrality bin of $\mathrm{Pb}-\mathrm{Pb}$ collisions. The FOS background is shown in (a). The ratio between FOS and $2 \sqrt{N^{++} N^{--}}$background, demonstrating the consistency between the two methods, is presented in (b). The signal spectra (c-d) are obtained by subtracting the combinatorial background spectra from the total opposite-sign dimuon mass distributions.

have then been tracked through the experimental set-up and, for the accepted events, the kinematical variables have been reconstructed using the procedure applied to the measured data. The ratio $A^{i}$ between the number of reconstructed and generated events, for a given process and in a certain phase space region, defines the acceptance of the apparatus for the process $i$ in that region. For $\mathrm{p}-\mathrm{W}$ collisions, in the analysis domain $\mathcal{D}^{p A}$, defined by the cuts $-0.52 \leq y_{\mathrm{cm}}<0.48,-0.5<\cos \theta<0.5$ and $M>1.5 \mathrm{GeV} / \mathrm{c}^{2}$, we get $A^{D Y}=6.2 \%, A^{D \bar{D}}=2.1 \%, A^{\psi}=14.4 \%$ and $A^{\psi^{\prime}}=16.8 \%$.

Having evaluated the shapes of the expected contributions it is possible to fit each of the p-A mass spectra with the function (4), leaving as free parameters the quantities $n^{i}$ (with $i=D Y, D \bar{D}, \psi, \psi^{\prime}$ ). Since the p-A Drell-Yan and open charm cross sections are both experimentally known $[25,26,27]$ to scale as $\mathrm{A}^{\alpha}$, with $\alpha=1$, we have imposed the same $\sigma_{D \bar{D}} / \sigma_{D Y}$ ratio for all the four $\mathrm{p}-\mathrm{A}$ systems. A priori, this 
assumption could be verified by fitting independently this ratio on each of the four p-A spectra, but our data are not sensitive enough to clearly recognize some specific trend in the dependence of $\sigma_{D \bar{D}} / \sigma_{D Y}$ with A. This is probably due to various sources of systematic uncertainties, which will be discussed in detail below. Consequently, we have performed a simultaneous fit of the four $\mathrm{p}-\mathrm{A}$ data samples in the mass region $M>1.5 \mathrm{GeV} / \mathrm{c}^{2}$ imposing a constant value for the ratios

$$
\left(\frac{D \bar{D}}{D Y}\right)_{i}=\frac{\left(n_{i}^{D \bar{D}} \int \frac{\mathrm{d} N_{i}^{D \bar{D}}}{\mathrm{~d} M} \mathrm{~d} M\right) / A_{i}^{D \bar{D}}}{\left(n_{i}^{D Y} \int \frac{\mathrm{d} N_{i}^{D Y}}{\mathrm{~d} M} \mathrm{~d} M\right) / A_{i}^{D Y}}
$$

where $i=\mathrm{p}-\mathrm{Al}, \mathrm{p}-\mathrm{Cu}, \mathrm{p}-\mathrm{Ag}, \mathrm{p}-\mathrm{W}$. The quantities $(D \bar{D} / D Y)_{i}$ are the acceptance corrected ratios between $D \bar{D}$ and $D Y$ dimuon production in the phase space domain $\mathcal{D}^{p A}$ defined above. The 4 measured p-A mass spectra can be simultaneously described by the function Eq. (4), with an overall constant value for $D \bar{D} / D Y$; furthermore, the result does not change (within $2 \%$ ) if the starting point for the fit is pushed down to $M=1.3 \mathrm{GeV} / \mathrm{c}^{2}$. In Fig. 5, we show the result of the simultaneous fit in the region $M>1.3 \mathrm{GeV} / \mathrm{c}^{2}$. It would have been possible to improve the description of the data in the charmonia mass region by adjusting the resonance shapes with more

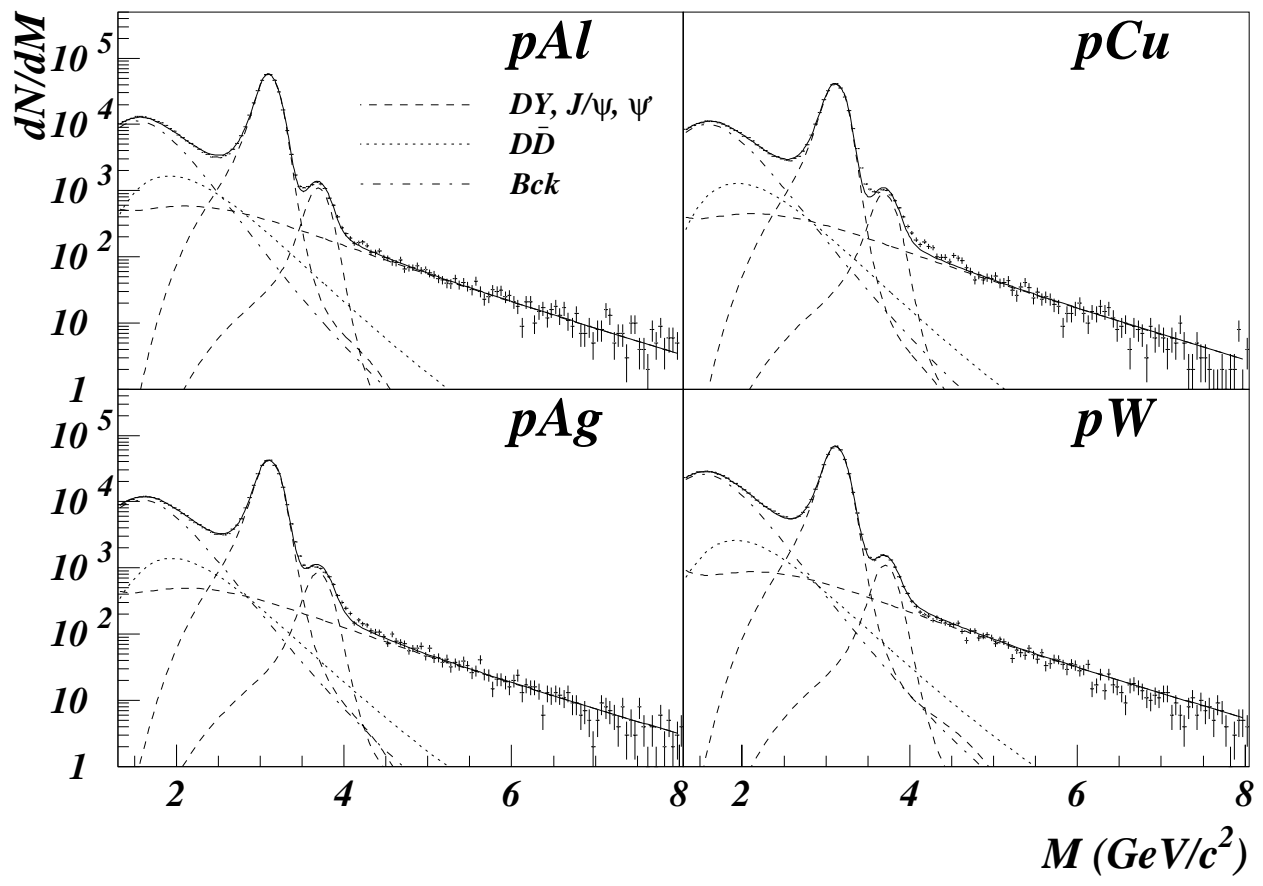

Figure 5: Simultaneous fit of the $4 \mathrm{p}$-A opposite-sign mass distributions in the mass range $M>1.3 \mathrm{GeV} / \mathrm{c}^{2}$. The dashed lines represent the $D Y, \mathrm{~J} / \psi$ and $\psi^{\prime}$ contributions, the dotted line the $D \bar{D}$ process, while the dashed-dotted line is the combinatorial background. 
parameters. However, the analysis of the IMR is insensitive to such refinements. The IMR signal distribution, defined as the background subtracted opposite sign dimuon spectrum, is well reproduced by a sum of $D \bar{D}$ and $D Y$ dimuons, plus a small contribution due to the tail of the $\mathrm{J} / \psi$, as shown in Fig. 6 .

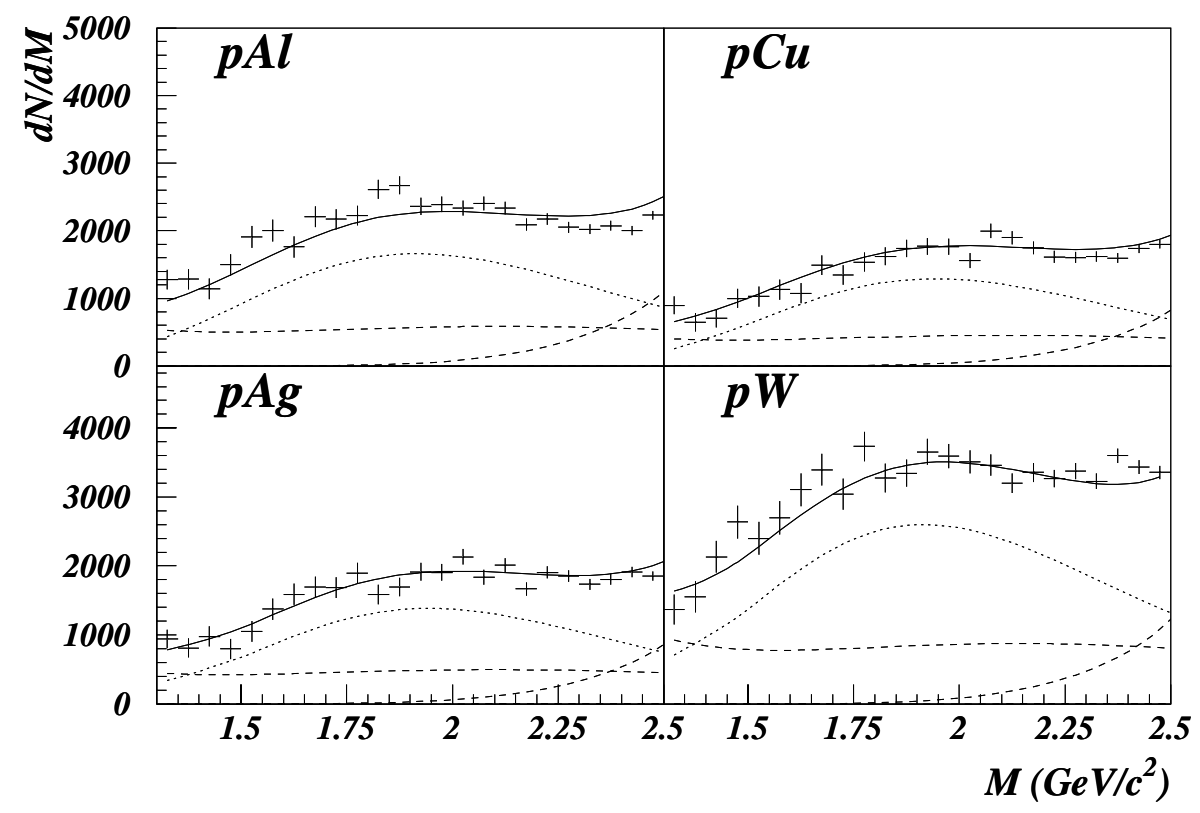

Figure 6: Background subtracted mass distributions in the IMR, compared with the sum of $D Y, D \bar{D}$ and $\mathrm{J} / \psi$ contributions, as resulting from the fit shown in Fig. 5 .

To evaluate the influence of the resonance region on the IMR results, we have performed a second fit, excluding the mass window $2.0<M<4.5 \mathrm{GeV} / \mathrm{c}^{2}$. We find that the quality of the fit improves (the $\chi^{2} /$ dof decreases from 3.4 to 1.6 ), but the $D \bar{D} / D Y$ ratio changes by only $\sim 6 \%$. In the fits described up to now, the combinatorial background contribution has been fixed, using the procedure described in Section 4. As a further test of the stability of the results, and in order to see if the normalization of the background could be constrained by the data, we have left the four $R$ factors as further free parameters in the fit (see Ref. [24] for details). The $D \bar{D} / D Y$ value changes by less than $1 \%$ and the $R$ factors are equal, within errors, to the ones of Table 3. Finally, we have estimated that the $D \bar{D} / D Y$ ratio is affected by $\mathrm{a} \sim 20 \%$ uncertainty due to differences between the simulated and measured $\mathrm{d} N / \mathrm{d} M$ shapes. This only affects the p-A runs, where the data taking conditions imposed by the very high beam intensity are not fully reproduced by the simulation procedures.

The $D \bar{D} / D Y$ value resulting from this data analysis is

$$
\left(\frac{D \bar{D}}{D Y}\right)_{p A, 450}=4.2 \pm 0.9
$$

where the error bar is dominated by the systematical uncertainties. 
Since we have verified that the p-A IMR mass spectrum can indeed be described as a superposition of Drell-Yan and open charm dimuons, we expect this to be true for the distributions of any other kinematical variable of the IMR dimuons. Therefore, we have compared the background subtracted $p_{\mathrm{T}}, y$ and $\cos \theta_{C S}$ distributions of the IMR events with a superposition of the expected $D Y$ and $D \bar{D}$ shapes, as calculated by PYTHIA, with the parameter values shown above. The contribution of the lower tail of the $\mathrm{J} / \psi$ has also been taken into account, and the relative normalizations of the various sources have been fixed to the values obtained in the fit of the mass spectra. Figure 7 shows a reasonably good agreement between the measured $p_{\mathrm{T}}, y$ and $\cos \theta_{C S}$ distributions of IMR dimuons and the sum of the expected sources, for the $\mathrm{p}-\mathrm{Al}$ data sample. The agreement is particularly remarkable for the $p_{\mathrm{T}}$ spectrum,
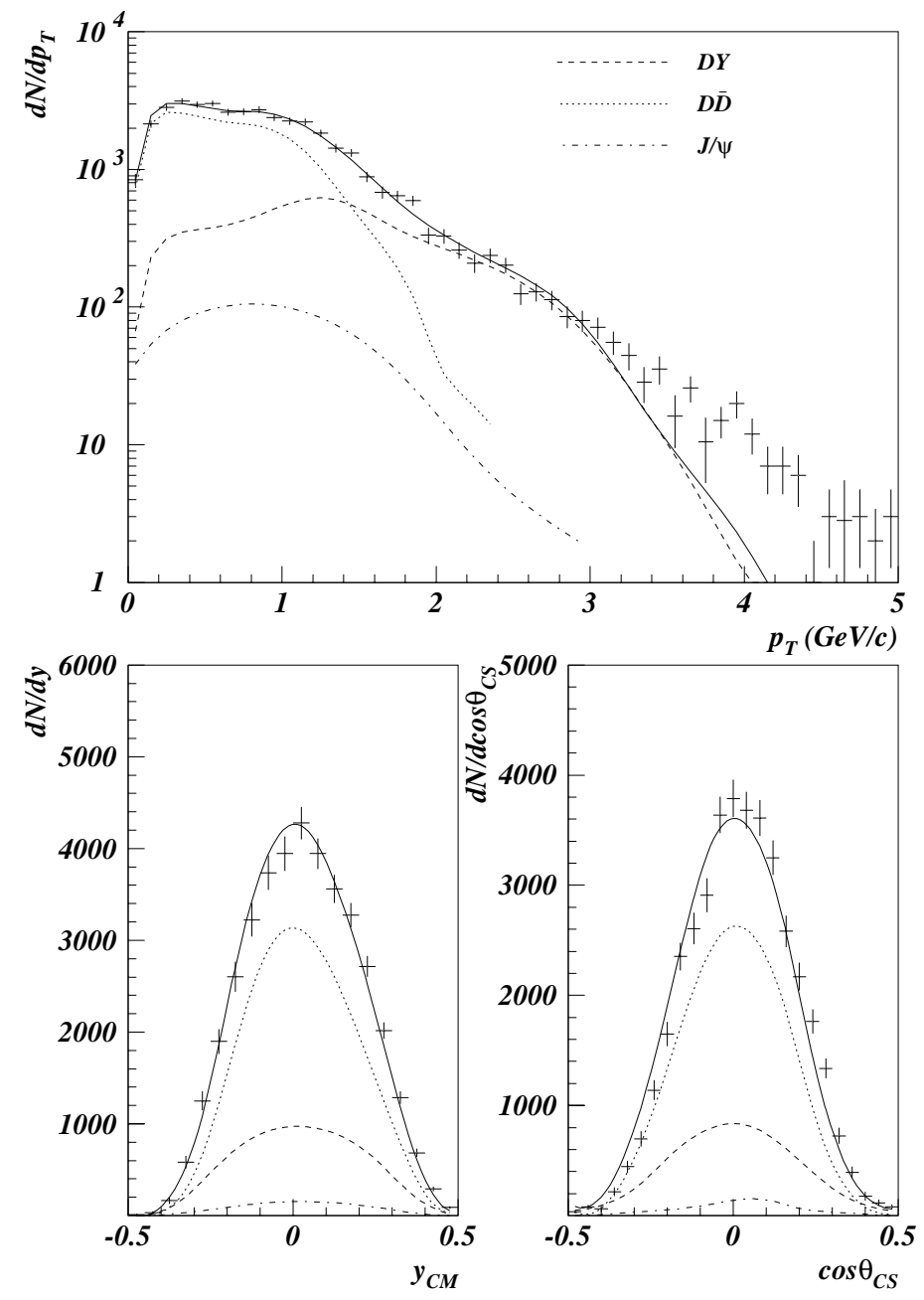

Figure 7: $p_{\mathrm{T}}, y$ and $\cos \theta_{C S}$ differential distributions of the IMR dimuons $(1.5<$ $M<2.5 \mathrm{GeV} / \mathrm{c}^{2}$ ), for the $\mathrm{p}-\mathrm{Al}$ system. The curves, calculated with PYTHIA, do not include NLO QCD diagrams, a possible explanation for the discrepancy visible for $p_{\mathrm{T}}$ around $4 \mathrm{GeV} / \mathrm{c}$. 
at least in the region up to $3 \mathrm{GeV} / \mathrm{c}$, since the various contributions have different expected shapes as a function of this variable. Therefore the study of the transverse momentum spectra can allow to disentangle the known sources, confirming in this case the result obtained with the study of the mass spectra. This is only partially true for the study of the $y$ and $\cos \theta_{C S}$ distributions since, with respect to these variables, all the expected sources have similar shapes in our acceptance.

\subsection{Determination of $\sigma_{c \bar{c}}$}

We have shown in the previous section that the contribution from semi-leptonic decays of charmed particles is quantitatively important in the IMR and we have determined its yield, with respect to the Drell-Yan process. To complete the analysis of the $D \bar{D}$ contribution to dimuon production in $\mathrm{p}-\mathrm{A}$ collisions, we will now derive the value of the total charm production cross section in pp collisions, $\sigma_{c \bar{c}}^{p p}$, that provides the best reproduction of our dimuon mass distributions.

It has been verified [28] that the event generator PYTHIA gives a reasonable description of the differential dilepton spectra from semi-leptonic decays of charmed mesons. Also the $\sqrt{s}$ dependence of the $D$-meson production cross sections is well reproduced. The absolute production cross sections, however, are underpredicted by PYTHIA, by a constant factor whose numerical value depends on the specific settings used in the calculation, as the mass of the $c$ quark or the choice of the $Q^{2}$ scale (see Ref. [28] for further details). Therefore, we can use PYTHIA to calculate the ratio between the total number of generated events (corresponding to a certain charm production cross section) and the number of $\mu^{+} \mu^{-}$pairs from simultaneous semileptonic decays of charmed mesons, in the kinematical window $\mathcal{D}^{p A}$ where the analysis of the p-A data is performed. This calculated ratio includes a weighted mean of the branching ratios for the decay of charmed mesons into muons [29].

From the measured $D \bar{D} / D Y$ ratio, and from the number of Drell-Yan events, we obtain the total number of muon pairs from $D \bar{D}$ decays needed to reproduce our $\mathrm{p}-\mathrm{A}$ data. This number can be translated into barn knowing the luminosity, which we have evaluated using the fitted number of $\mathrm{J} / \psi$ events and the previously measured $\mathrm{J} / \psi$ production cross sections in $\mathrm{p}-\mathrm{W}$ collisions [30, 31]. Finally, to compare the measured $\mathrm{p}$-A values with the calculated pp values we have assumed a linear dependence of the open charm cross section on the target mass number, $A$. This way we derive the total open charm production cross section, in pp collisions at $450 \mathrm{GeV} / \mathrm{c}$, as

$$
\sigma_{c \bar{c}}^{p p}=\left(\frac{\sigma_{c \bar{c}}^{p p}}{\sigma_{D \bar{D} \rightarrow \mu^{+} \mu^{-}}^{p p}}\right)^{\text {PYTHIA }} \cdot \frac{\sigma_{D \bar{D} \rightarrow \mu^{+} \mu^{-}}^{p A \text { data }}}{A}=36.2 \pm 9.1 \mu \mathrm{b}
$$

The error bar is dominated by the uncertainty on the branching ratios. In Fig. 8 we compare our indirect measurement of $\sigma_{c \bar{c}}^{p p}$ (divided by 2 to consider only the $x_{F}>0$ hemisphere) with direct measurements of the charm hadroproduction cross section, made by several other experiments. The agreement is very satisfactory, supporting 
the hypothesis that intermediate mass dimuon production in $\mathrm{p}-\mathrm{A}$ collisions is properly described by a superposition of Drell-Yan dimuons and muon pairs from decays of charmed hadrons.

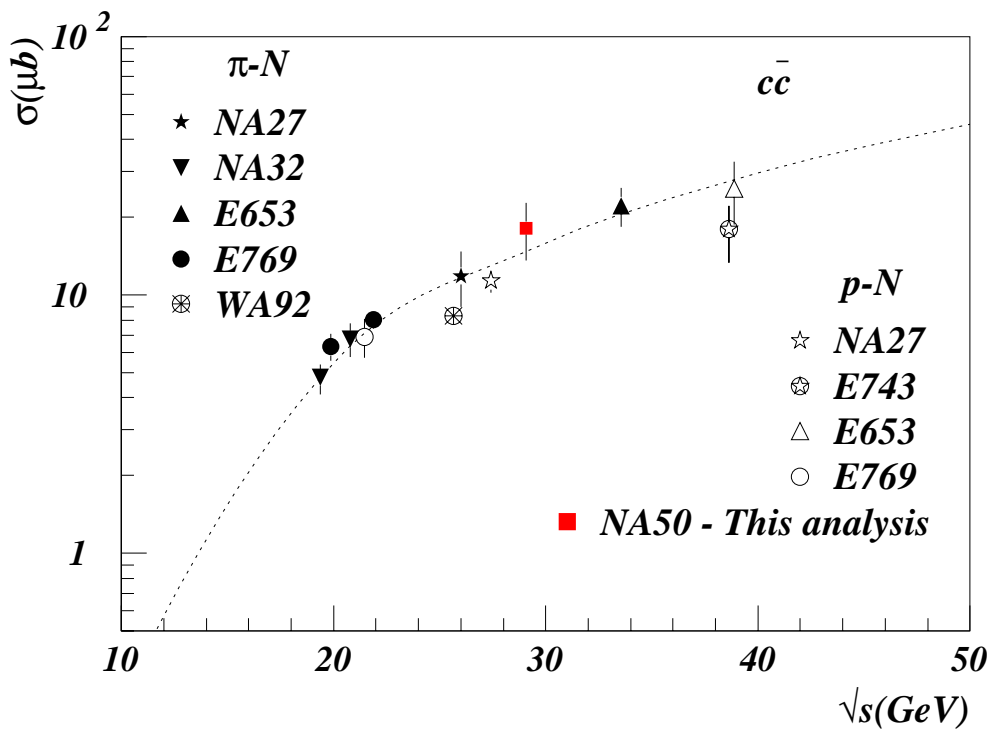

Figure 8: Compilation of charm hadroproduction cross section measurements (from [28] and references therein). The values refer to forward $\left(x_{F}>0\right)$ production. The results obtained with nuclear targets have been divided by the target mass number, $A$. The dotted curve shows the $\sqrt{s}$ - dependence of $\sigma_{c \bar{c}}^{p p}$ obtained with PYTHIA, fitted to the experimental points.

\section{3 $\mathrm{S}-\mathrm{U}$ and $\mathrm{Pb}-\mathrm{Pb}$ collisions}

As a first step in the IMR study of nucleus-nucleus collisions we will try to describe the $200 \mathrm{GeV} /$ nucleon $\mathrm{S}-\mathrm{U}$ and $158 \mathrm{GeV} /$ nucleon $\mathrm{Pb}-\mathrm{Pb}$ mass spectra performing a linear extrapolation of the dimuon sources already present in $\mathrm{p}-\mathrm{A}$. The evaluation of the $D \bar{D}$ contribution to the nucleus-nucleus spectra is performed starting from $\sigma_{c \bar{c}}^{p p}$, calculated at the relevant energies. The values are obtained using the $\sqrt{s}$-dependence of $\sigma_{c \bar{c}}$ calculated by PYTHIA, normalized to match the value of $\sigma_{c \bar{c}}^{p p}, 450$ derived from the NA50 p-A data. The nuclear dependence of the cross section is parametrized assuming an $A_{p} \times A_{t}$ dependence (where $A_{p}$ and $A_{t}$ are the mass numbers of the projectile and target, respectively) as is expected for a hard process. Then, we calculate the cross section for the production of charmed mesons that decay into muons in the kinematical domain $\mathcal{D}^{A B}$ where the analysis is performed $\left(0<y_{\mathrm{cm}}<1\right.$, $\left.-0.5<\cos \theta_{C S}<0.5, M>1.5 \mathrm{GeV} / \mathrm{c}^{2}\right)$. We obtain $\sigma_{c \bar{c} \rightarrow \mu^{+} \mu^{-}}^{S U \text {,expected }}=2.7 \pm 0.6 \mu \mathrm{b}$ and $\sigma_{c \bar{c} \rightarrow \mu^{+} \mu^{-}}^{P b P \text {,exped }}=9.6 \pm 2.3 \mu \mathrm{b}$. From these quantities it is possible to calculate the expected number of $D \bar{D}$ dimuons in the centrality integrated $\mathrm{S}-\mathrm{U}$ and $\mathrm{Pb}-\mathrm{Pb}$ 
spectra, as well as the ratio $(D \bar{D} / D Y)^{\text {expected }}$. To estimate such ratio we use the measured $D Y$ events, as determined by a fit to the high mass region of the spectrum. We get $(D \bar{D} / D Y)_{S U}^{\text {expected }}=1.29 \pm 0.14$ and $(D \bar{D} / D Y)_{P b P b}^{\text {expected }}=1.04 \pm 0.08$. These values are considerably lower than the ones quoted for $\mathrm{p}$-A, because of the different $\sqrt{s}$ dependence of $\sigma_{c \bar{c}}$ and $\sigma_{D Y}$.

In nucleus-nucleus collisions, the Drell-Yan production cross-section, scales with $A_{p} \times A_{t}$ when integrated over impact parameter [26], as expected from a hard process proportional to the number of elementary nucleon-nucleon collisions. The same argument holds for open charm production so that, for a given system, the ratio $(D \bar{D} / D Y)^{\text {expected }}$ should be a constant, independent of the considered centrality bin. We can then perform a fit of the measured mass spectra in the high mass region $\left(M>2.9 \mathrm{GeV} / \mathrm{c}^{2}\right)$, to fix the $D Y$ component, and calculate for each bin $i$ the expected number of $D \bar{D}$ dimuons as

$$
N_{D \bar{D}, i}^{\text {expected }}=\left(\frac{D \bar{D}}{D Y}\right)^{\text {expected }} \cdot N_{D Y, i}^{\text {measured }}
$$

With this procedure we find that the sum of the expected sources systematically underestimates the IMR data. As an example we show in Fig. 9 the result for a peripheral and a central $\mathrm{Pb}-\mathrm{Pb}$ bin.
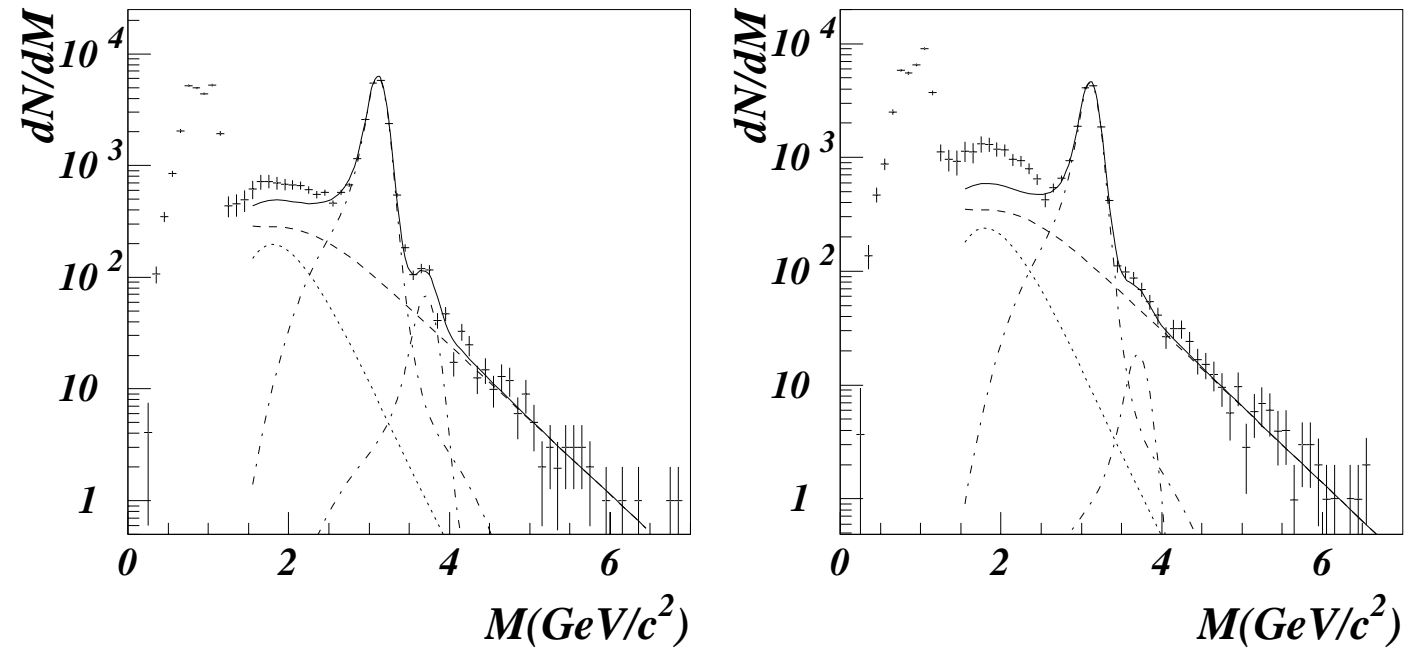

Figure 9: Comparison between data and the sum of expected sources (solid line) for a peripheral $\left(\left\langle N_{\text {part }}\right\rangle=110\right)$ and a central $\left(\left\langle N_{\text {part }}\right\rangle=381\right) \mathrm{Pb}-\mathrm{Pb}$ bin. The $D Y$ (dashed line), $\mathrm{J} / \psi, \psi^{\prime}$ (dashed-dotted line) and $D \bar{D}$ (dotted line) contributions are also shown. The excess in the IMR is clearly visible.

More generally, the excess in the IMR grows with centrality for both S-U and $\mathrm{Pb}-\mathrm{Pb}$ collisions. This can be seen in Fig. 10 where we show the quantity $D / S$, defined as the ratio between the measured dimuon signal and the sum of the expected sources $\left(D \bar{D}, D Y\right.$ and $\mathrm{J} / \psi$ tail) in the mass region $1.5<M<2.5 \mathrm{GeV} / \mathrm{c}^{2}$, versus 
the number of participant nucleons $N_{\text {part }}$ (calculated using the model described in Ref. [16]). Note that the direct comparison between the values of $D / S$ obtained for $\mathrm{S}-\mathrm{U}$ and for $\mathrm{Pb}-\mathrm{Pb}$ is not meaningful since, because of the different values of the magnetic field in the spectrometer for the two set-ups, the acceptances may be different for the a priori unknown physical process responsible for the excess.
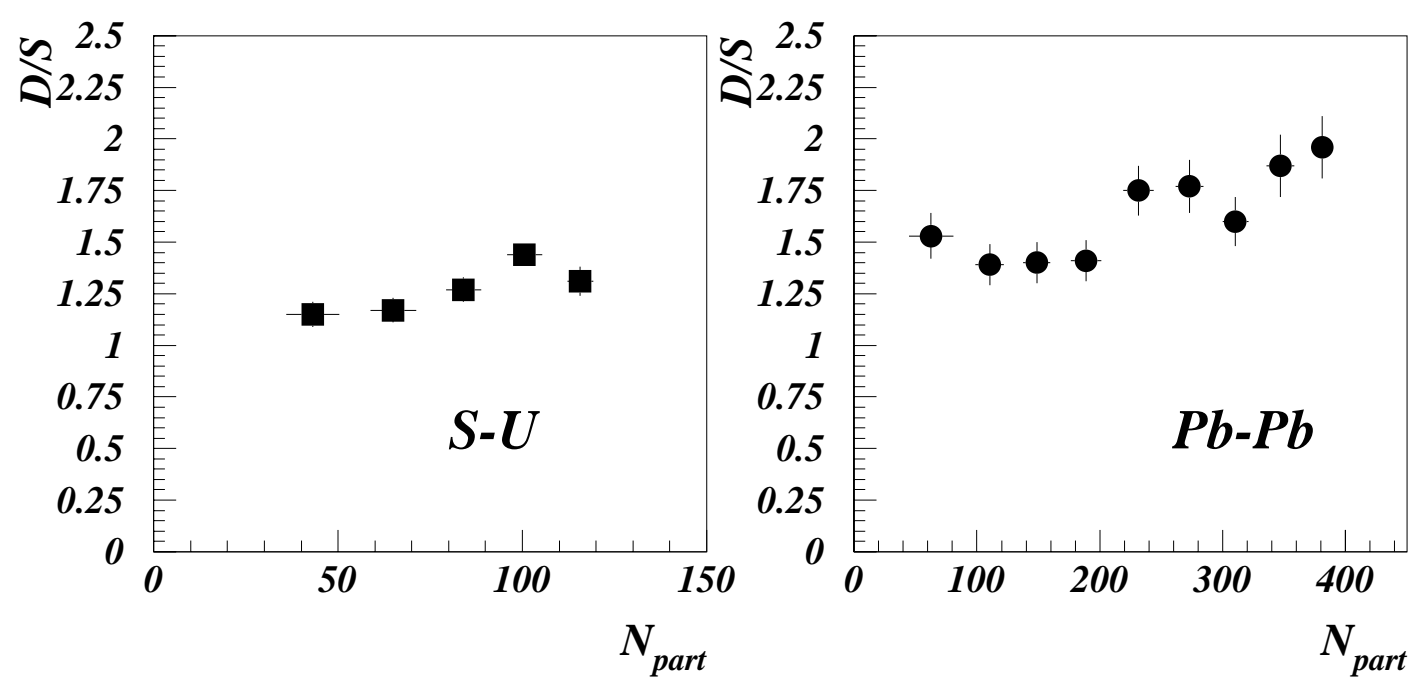

Figure 10: The ratio $D / S$ (data over expected sources) as a function of the number of participant nucleons.

\subsection{Nature of the excess}

Having established the presence of an IMR excess in nucleus-nucleus collisions, we can test against the data some tentative explanations of its origin. The two simplest hypotheses are that the excess is due to an enhancement of the $D \bar{D}$ component, or to a wrong evaluation of the normalization of the combinatorial background.

To test these hypotheses we fit the IMR excess mass spectra using as fitting function either the $D \bar{D}$ or the background shape, leaving in each fit the normalization constant as a free parameter. In order to increase the statistical significance of the result we have used a reduced set of centrality intervals, merging the bins defined in Table 1. The results are shown in Fig. 11 for $\mathrm{Pb}-\mathrm{Pb}$. The mass distribution of the excess appears to be compatible with the one foreseen for open charm decays $\left(\chi^{2} /\right.$ dof $\left.\sim 1\right)$ and significantly different from the background shape $\left(\chi^{2} /\right.$ dof $\left.\sim 3\right)$.

To quantify a possible enhancement of the $D \bar{D}$ component, we have fitted all the $\mathrm{S}-\mathrm{U}$ and the $\mathrm{Pb}-\mathrm{Pb}$ opposite sign mass spectra in the region $M>1.5 \mathrm{GeV} / \mathrm{c}^{2}$ with the function (4), leaving free in the fit all the normalization constants and keeping as usual the background contribution fixed. All the mass spectra are satisfactorily described, i.e. we can account for IMR dilepton production in heavy-ion collisions by means of a simple superposition of $D Y$ and open charm decay muons, as in the p-A 


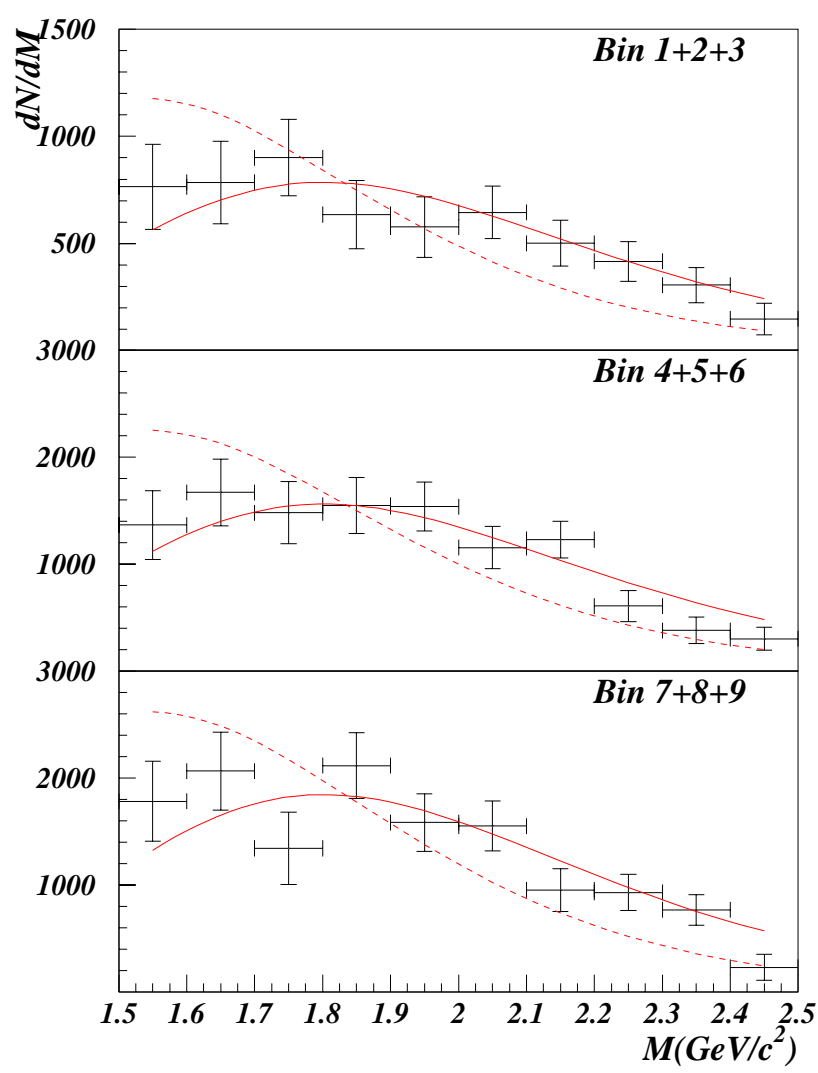

Figure 11: Comparison of the excess mass distribution with the open charm (solid line) and background (dashed line) shapes.

case. The $D \bar{D} / D Y$ values obtained in the fits range from $1.62 \pm 0.15$ to $2.42 \pm 0.13$ for $\mathrm{S}-\mathrm{U}$, and from $2.05 \pm 0.25$ to $3.71 \pm 0.39$ for $\mathrm{Pb}-\mathrm{Pb}$. When compared to the expected values $(D \bar{D} / D Y)_{S U(P b P b)}^{\text {expected }}$, derived in the previous section, they immediately give the enhancement factor $E$ of the open charm production yield. Figure 12 shows $E$ as a function of the number of participants, $N_{\text {part }}$. The numerical values are listed in Table 4. The quoted errors on the nucleus-nucleus points are dominated by the statistical uncertainty. We observe that, in order to describe the data with a simple superposition of $D Y$ plus $D \bar{D}$, the expected open charm yield has to be scaled up by a factor that increases roughly linearly with $N_{\text {part }}$, reaching a factor $\sim 3.5$ for central $\mathrm{Pb}-\mathrm{Pb}$ collisions.

The hypothesis that the excess dimuons are due to charm enhancement can be further tested studying the $p_{\mathrm{T}}, y$ and $\cos \theta_{C S}$ distributions of the nucleus-nucleus intermediate mass dimuons. We use a procedure analogue to the one described in Section 5.1, i.e. we compare the background subtracted spectra with a superposition of $D Y, D \bar{D}$ and $\mathrm{J} / \psi$ dimuons, with relative normalizations taken from the results of the fits to the IMR mass spectra. The differential distributions of the dimuon sources have again been calculated with the PYTHIA code, using the set of initial 


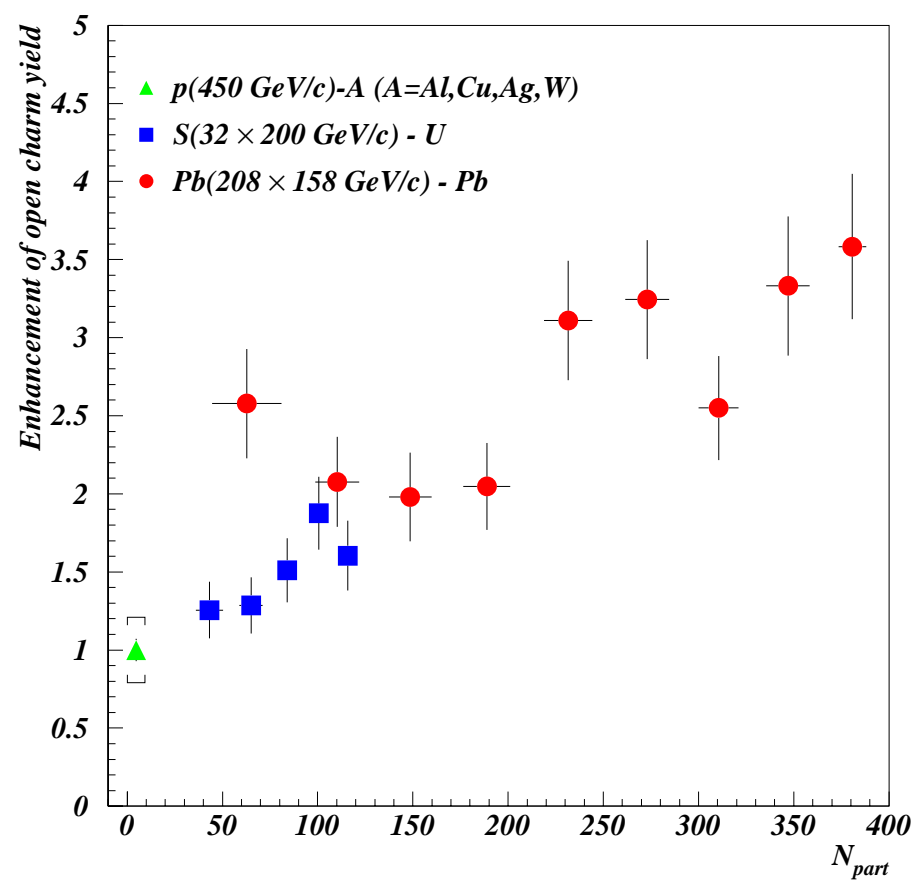

Figure 12: The enhancement $E$ of the open charm yield needed to describe the measured IMR data, as a function of the number of participants. The bracket in the p-A point represents the systematical uncertainty.

\begin{tabular}{rrc}
\hline & $\left\langle N_{\text {part }}\right\rangle$ & $E_{\text {open-charm }}$ \\
\hline $\mathrm{p}-\mathrm{W}$ & $5 \pm 2$ & 1.0 \\
\hline & $43 \pm 7$ & $1.26 \pm 0.18$ \\
& $65 \pm 6$ & $1.29 \pm 0.18$ \\
$\mathrm{~S}-\mathrm{U}$ & $84 \pm 5$ & $1.51 \pm 0.20$ \\
& $101 \pm 5$ & $1.88 \pm 0.23$ \\
$116 \pm 3$ & $1.61 \pm 0.22$ \\
\hline & $63 \pm 18$ & $2.58 \pm 0.35$ \\
$110 \pm 11$ & $2.08 \pm 0.29$ \\
$149 \pm 11$ & $1.98 \pm 0.28$ \\
$189 \pm 12$ & $2.05 \pm 0.28$ \\
$\mathrm{~Pb}-\mathrm{Pb}$ & $231 \pm 13$ & $3.11 \pm 0.38$ \\
& $273 \pm 11$ & $3.24 \pm 0.38$ \\
& $310 \pm 10$ & $2.55 \pm 0.33$ \\
& $347 \pm 11$ & $3.33 \pm 0.45$ \\
$381 \pm 7$ & $3.58 \pm 0.46$ \\
\hline
\end{tabular}

Table 4: Enhancement of the open-charm yield needed to describe the measured IMR dimuon yield. 
parameters (charm quark mass, parton intrinsic $k_{T}$ ) already adopted for the study of p-A collisions. In Fig. 13 we show the IMR $p_{\mathrm{T}}$ distribution for a peripheral and a central $\mathrm{Pb}-\mathrm{Pb}$ bin. The $D \bar{D}$ contribution shown in these figures includes the enhanced normalization fixed from the analysis of the mass spectra, leading to a reasonable description of the data. The analysis of the $y$ and $\cos \theta_{C S}$ spectra is less conclusive, since all the contributions have similar shapes in the acceptance window of the apparatus. Anyway, a good agreement with the data is found, as can be seen in Fig. 14, where we show the $y$ and $\cos \theta_{C S}$ distributions of the IMR dimuons, compared with the $D Y, J / \psi$ and enhanced $D \bar{D}$ contributions, for a peripheral $\mathrm{Pb}-\mathrm{Pb}$ bin.
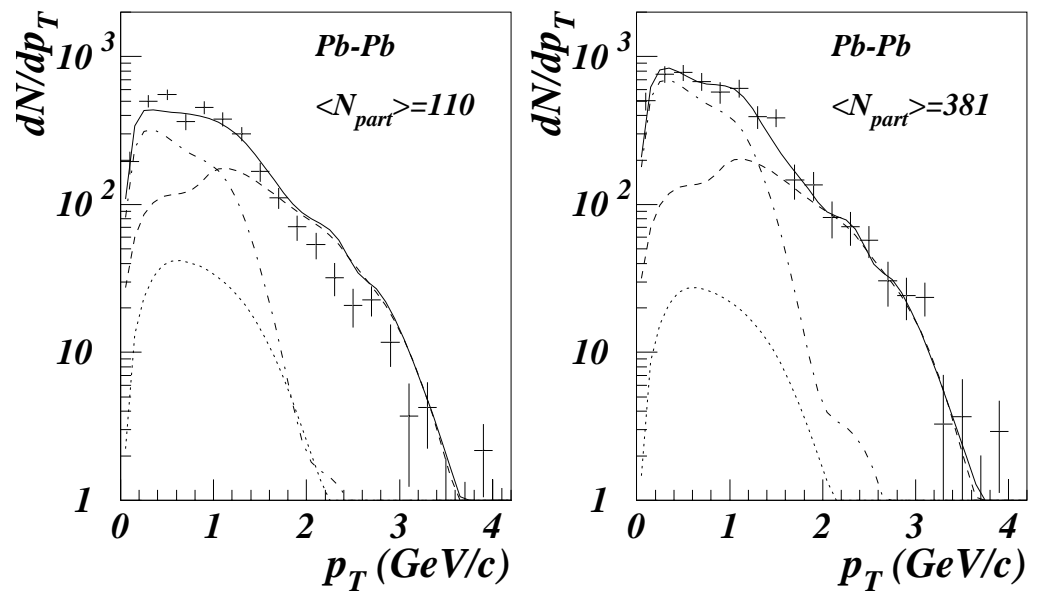

Figure 13: The $p_{\mathrm{T}}$ distribution of IMR dimuons for peripheral (left) and central (right) $\mathrm{Pb}-\mathrm{Pb}$ collisions. The $D Y$ (dashed line), $\mathrm{J} / \psi$ (dotted line) and $D \bar{D}$ (dashed-dotted line) contributions are shown, as well as their sum (solid line).
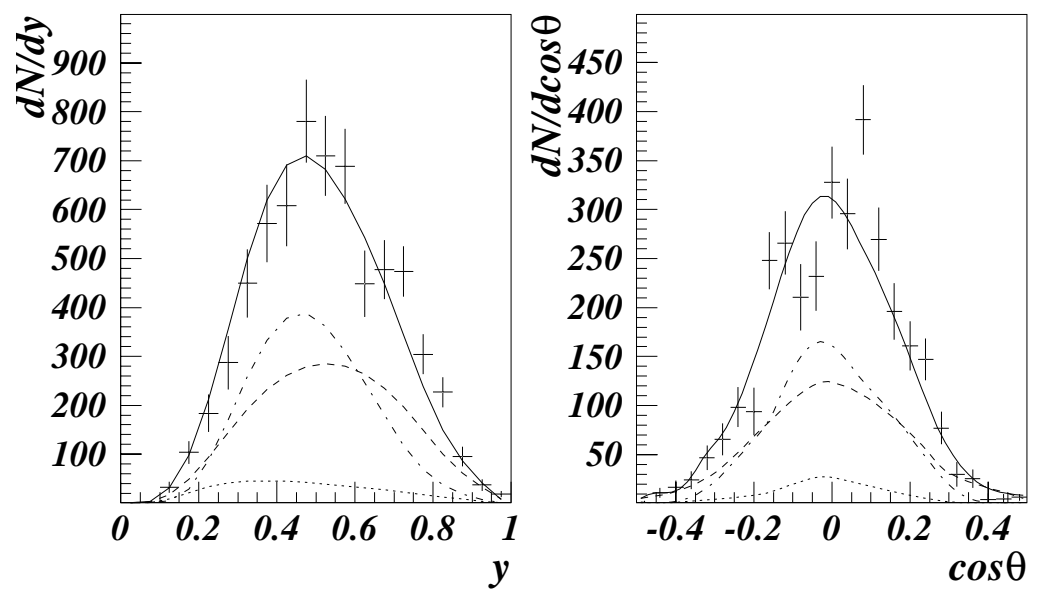

Figure 14: The $y$ and $\cos \theta_{C S}$ distributions of IMR dimuons for a peripheral $\mathrm{Pb}-\mathrm{Pb}$ bin $\left(\left\langle\mathrm{N}_{\text {part }}\right\rangle=110\right)$. The $D Y$ (dashed line), $\mathrm{J} / \psi$ (dotted line) and $D \bar{D}$ (dashed-dotted line) contributions are shown, as well as their sum (solid line). 


\subsection{Discussion}

The analysis presented in this paper shows that the measured yields of IMR dimuons, in nucleus-nucleus collisions, cannot be reproduced by the expected dimuon sources, if their contribution is fixed by a linear extrapolation of the p-A results. Secondly, the differential shapes of the excess events are compatible with the open charm source. We will now discuss these two issues separately.

Concerning the existence of an excess, the most delicate point to discuss is the combinatorial background subtraction. Its contribution being so high, the subtraction procedure is crucial, in particular the determination of the normalization factor $R$, which has been obtained through a Monte-Carlo simulation. We have already seen that in p-A collisions the simulation gives a result in very good agreement with a measured value of $R$; moreover, when we leave the background normalization free in the fit of the high statistics p-A data, we are led to values of $R$ in agreement with the calculated ones. On the other hand, an event generator which correctly estimates the charge correlation effects in $\mathrm{p}-\mathrm{A}$, could a priori not do the same proper job for nucleus-nucleus collisions. Therefore, we have performed some further tests on the influence of the choice of $R$ on our results. First we consider the possibility that, due to the high hadron multiplicities of nucleus-nucleus interactions, the charge correlation effects are negligible and $R=1$ everywhere. This choice had been adopted in older studies of the IMR $[3,6]$. The effect of this choice on the excess is negligible for the central bins, where also the Monte-Carlo calculation gives $R=1$, while for the peripheral ones the excess is increased, by about $20 \%$. The results obtained with $R=1$ are qualitatively comparable with the ones quoted in Refs. [3, 6], derived from lower statistics data samples. As a second check we have tried to fit the invariant mass spectra leaving $R$ as a free parameter, to see if the data alone could constrain the background normalization. In this case the $R$ factors for S-U decrease and become lower than unity, while for $\mathrm{Pb}-\mathrm{Pb}$ they slightly increase. This shows that for nucleusnucleus collisions the data alone cannot constrain in a consistent way the $R$ factors. However, it is clear that $R$ can be artificially raised up to some higher value, leaving no place for the excess. In this extreme case we have verified, by fixing the other contributions to their expected value and leaving the background normalization free, that we are no longer able to fit the data satisfactorily. For the most central $\mathrm{Pb}-\mathrm{Pb}$

bin, for example, the $\chi^{2}$ of the fit grows by about a factor 5 in the IMR. This reflects the fact that the excess does not have the shape of the combinatorial background (see Fig. 11). We conclude, therefore, that the presence of an IMR excess which increases with centrality, has been established unambiguously.

Concerning the interpretation of the excess, we have found that its mass and transverse momentum distributions are consistent with the ones we have calculated for the semileptonic decays of $D$-meson pairs. The same open charm distributions have been found to describe satisfactorily the p-A data. These two facts indicate that the origin of the excess may be an enhancement of the charm yield. On the other hand, it must be noted that any dimuon source which happens to give, in the 
acceptance of the spectrometer, differential distributions similar to the ones calculated for open charm, should be taken as an equally probable source of the observed excess. Theoretical work on this subject is presently in progress, and alternative explanations to open charm enhancement have been proposed, including the production of thermal dimuons [32, 33, 34], or rescattering of $D$-mesons in nuclear matter [35], which may lead to a local enhancement in the acceptance of the spectrometer. Further work along these lines is being carried out inside the collaboration and will be the subject of future publications. In order to discriminate between the various scenarios, a direct open charm measurement in nucleus-nucleus collisions would be welcome.

\section{Conclusions}

We have presented in this paper the analysis of the intermediate mass region of the dimuon spectrum measured in $\mathrm{p}-\mathrm{A}, \mathrm{S}-\mathrm{U}$ and $\mathrm{Pb}-\mathrm{Pb}$ collisions, by the NA38 and NA50 collaborations. The IMR p-A data can be described as a superposition of $D Y$ and $D \bar{D}$ dimuons, using PYTHIA to calculate the expected differential spectra for the two contributions. A good description of the p-A data is obtained assuming $\sigma_{c \bar{c}}^{p p}=36.2 \pm 9.1 \mu \mathrm{b}$ for $\sqrt{s}=29 \mathrm{GeV}$. A linear extrapolation of the p-A sources underestimates the nucleus-nucleus data by an average factor $\sim 1.27$ for $\mathrm{S}-\mathrm{U}$ and $\sim 1.65$ for $\mathrm{Pb}-\mathrm{Pb}$ collisions. The dimuon excess increases with the number of nucleons participating in the collision. Although alternative explanations cannot, at this moment, be excluded, we find that we can quantitatively explain the data assuming that open charm production is abnormally enhanced in nucleus-nucleus collisions. The enhancement factor increases linearly with the number of participant nucleons, up to a value 3.5 for central $\mathrm{Pb}-\mathrm{Pb}$ interactions. The differential distributions of the excess are also consistent with the shapes calculated for open charm decays.

\section{References}

[1] For a recent review see L. Kluberg, in: Proc. 14th Int. Conf. on Ultrarelativistic Nucleus-Nucleus Collisions, Quark Matter '99, Torino, Italy, May 1999, Nucl. Phys. A 661 (1999) 300c.

[2] See, for instance, P.V. Ruuskanen, Nucl. Phys. A544 (1992) 169c.

[3] C. Lourenço et al. (NA38 Coll.), Nucl. Phys. A566 (1994) 77c.

[4] A. Borhani, PhD Thesis, Université Pierre et Marie Curie, Paris, 1996.

[5] A.L.S. Angelis et al. (HELIOS/3 Coll.), preprint CERN-EP/98-92, Euro. Phys. J. C, in print.

[6] E. Scomparin et al. (NA50 Coll.), Nucl. Phys. A610 (1996) 331c.

[7] M.C. Abreu et al. (NA38 Coll.), Phys. Lett. B220 (1989) 471.

[8] L. Anderson et al., Nucl. Instrum. Meth. 223 (1984) 26.

[9] M.C. Abreu et al. (NA50 Coll.), Phys. Lett. B410 (1997) 327. 
[10] F. Bellaiche et al., Nucl. Instrum. Meth. A398 (1997) 180.

[11] B. Alessandro et al., Nucl. Instrum. Meth. A360 (1995) 189.

[12] R. Arnaldi et al., Nucl. Instrum. Meth. A411 (1998) 1.

[13] V. Capony, PhD Thesis, Université de Savoie, Annecy, 1996.

[14] S. Silva, PhD Thesis, Universidade Técnica de Lisboa, 1997.

[15] M.C. Abreu et al. (NA50 Coll.), Phys. Lett. B450 (1999) 456.

[16] F. Fleuret, PhD Thesis, Ecole Polytechnique, Palaiseau, 1997.

[17] K. Werner, Phys. Rep. 232 (1993) 87.

[18] M. Idzik et al. (NA50 Coll.), Proc. of the Multiparticle Dynamics International Conference 1999.

[19] S. Constantinescu et al., preprint IPNO-DRE-96-01.

[20] C. Lourenço, PhD Thesis, Universidade Técnica de Lisboa, 1995.

[21] S. Papillon, PhD Thesis, Université Paris-Sud, Orsay, 1991.

[22] T. Sjostrand, Computer Physics Commun. 82 (1994) 74.

[23] A.D. Martin et al., Phys. Rev. D51 (1995) 4756.

[24] C. Soave, PhD Thesis, Università di Torino, 1998.

[25] D.M. Alde et al. (E772 Coll.), Phys. Rev. Lett 64 (1990) 2479.

[26] M.C. Abreu et al. (NA50 Coll.), Phys. Lett. B410 (1997) 337.

[27] M.J. Leitch et al. (E789 Coll.), Phys. Rev. Lett. 72 (1994) 2542.

[28] P. Braun-Munzinger et al., Euro. Phys. J. C1 (1998) 123.

[29] C. Caso et al. (Particle Data Group), Euro. Phys. J. C3 (1998) 1.

[30] L. Fredj, PhD Thesis, Université Blaise Pascal, Clermont-Ferrand, 1991.

[31] M.C. Abreu et al. (NA38 Coll.), Phys. Lett. B449 (1999) 128.

[32] R. Rapp and E. Shuryak, hep-ph/9909348.

[33] K. Gallmeister et al., hep-ph/9908269.

[34] P. Huovinen et al., Nucl. Phys. A650 (1999) 227.

[35] Z. Lin et al., Phys. Lett. B444 (1998) 245. 